\title{
Traveling-wave thermoacoustic refrigerator driven by a multistage traveling-wave thermoacoustic engine
}

\author{
Esmatullah Maiwand Sharify*, Shinya Hasegawa \\ Department of Prime Mover Engineering, Tokai University, Kanagawa 259-1292, Japan
}

*Corresponding author. E-mail address: esmat.maiwand@gmail.com.

\begin{abstract}
A double-loop-type traveling-wave thermoacoustic refrigerator driven by a multistage traveling-wave engine was constructed. To reduce the onset temperature for thermoacoustic oscillations and achieve a refrigerator temperature of $-100{ }^{\circ} \mathrm{C}$, three etched stainless steel mesh regenerators were installed close to the sweet spot within the prime mover loop and one regenerator was fixed in the refrigerator loop. The maximum measured COP of the whole system at $50^{\circ} \mathrm{C}$, was 0.029 . Gas oscillations occurred when the hot temperature of the regenerator in the prime mover loop exceeded $85{ }^{\circ} \mathrm{C}$. On the other hand, refrigeration $\left(-42.3{ }^{\circ} \mathrm{C}\right)$ was observed when the hot heat exchanger temperature reached $90{ }^{\circ} \mathrm{C}$, which is lower than the boiling point of water. Furthermore, the refrigerator achieved a minimum cold temperature of $-107.4{ }^{\circ} \mathrm{C}$ when the hot temperature was $270{ }^{\circ} \mathrm{C}$.
\end{abstract}

Keywords: Thermoacoustic, Refrigerator, Traveling-wave, Multistage traveling-wave engine, Sweet spot, Etched stainless steel mesh regenerators

\section{Introduction}

In 1979, Ceperley ${ }^{1}$ proposed a traveling-wave thermoacoustic engine (TWTE) in which the acoustic power was amplified by traveling-wave propagation through a differentially heated regenerator. In a TWTE, when the phase difference $\Phi$ between the pressure and the cross-sectional mean velocity is zero and viscous losses are negligible, the gas parcels experience thermodynamically reversible processes. Hence, the efficiency of the engine ideally reaches the Carnot efficiency. The concept of Ceperley was practically realized by Yazaki et al. in $1998{ }^{2}$ They were the first to build a looped tube TWTE and observed that the energy conversion between heat flow and work flow was executed through the Stirling cycle.

In 1999, Backhaus and Swift ${ }^{3}$ built a prototype thermoacoustic engine based on traveling-wave energy conversion and introduced a resonator in a looped tube. They created the traveling-wave phasing with high acoustic impedance in the regenerator area, which is called the sweet spot. Accordingly, their engine efficiency reached above $30 \%$ thermal efficiency. The sweet spot is a location where the volume velocity is minimum and the phase difference between the pressure and velocity is zero. ${ }^{4}$ The sweet spot is dominant when an acoustic wave has high acoustic impedance, in particular when the dimensionless specific acoustic impedance $z$ is greater than or equal to 10 times the specific heat ratio $\gamma$ (i.e., $z \geq 10 \gamma$ ). In the same year, Swift et al. ${ }^{5}$ studied a pulse tube refrigerator with acoustic power recovery of 
lost power in orifice-type pulse tube cryocoolers, which is now called a traveling-wave thermoacoustic refrigerator (TWTR).

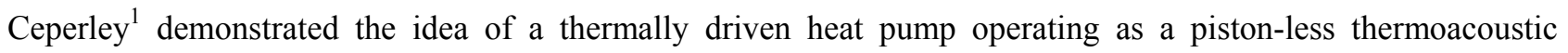
refrigerator, which consisted of a pair of TWTEs. Based on the idea of Ceperley, Yazaki et al. ${ }^{6}$ constructed a loopedtube thermoacoustic refrigerator driven by a TWTE. Their engine could not achieve high efficiency because of the relatively small value of $z$ with respect to $\gamma(z=3 \gamma)$. This problem was solved by Ueda and Yazaki ${ }^{7}$ using a resonator in a looped tube and adjusting the position of the regenerator close to the sweet spot. In their study, $z$ reached a value 10 times greater than $\gamma(z=10 \gamma)$. They achieved a cold temperature of around $-25^{\circ} \mathrm{C}$ with a cooling power of $11 \mathrm{~W}$. Yazaki et al..$^{6}$ used two regenerators in one loop to realize the functions of both a TWTE and TWTR, so the efficiency was still low because one loop does not have two sweet spots to simultaneously keep the pressure and velocity waves in phase. In 2006, Luo et al. ${ }^{8}$ built a thermoacoustic refrigerator with two separate traveling-wave loops, and obtained a lowest temperature of $-64.4{ }^{\circ} \mathrm{C}$. Furthermore, Yu et al. ${ }^{9}$ introduced and tested new configurations of TWTRs driven by a TWTE in 2011. They found that a TWTR with an assembly of lumped inertial mass and flexible membrane as the phase shifter improved the cooling performance.

The temperature of most industrial waste heat ranges from 400 to $600 \mathrm{~K}$, while the critical onset temperature of a thermoacoustic engine is generally in the range 600 to $1000 \mathrm{~K}^{3}$ To overcome this problem, Gardner and Swift ${ }^{4}$ proposed a multistage thermoacoustic engine that can lower the critical onset temperature and thereafter the multi-stage thermoacoustic systems were reported by many researcher. ${ }^{10-17}$ In 2010, Biwa revealed that the installation of multiple regenerators at suitable positions can markedly enhance acoustic power production, and they obtained a critical onset temperature ratio of $1.19 .{ }^{18}$ Although success has been reported in lowering the critical temperature using multiple regenerators, the appropriate positions of the regenerators and the tube radius to produce low-temperature oscillations with high efficiency have not been established. ${ }^{19}$ Therefore, further development of such engines is extremely important for many practical applications.

In 2013, Hasegawa et al. ${ }^{19}$ (one of the authors of this paper) numerically optimized a multistage-type thermoacoustic engine to produce thermoacoustic oscillations equivalent to a typical industrial waste heat temperature. They considered three regenerators at optimized positions close to the sweet spot within the prime mover loop, and consequently determined the configuration that enables a low temperature oscillation with high efficiency. Furthermore, they obtained the dependency on the temperature ratio of the prime mover for the temperature ratio of the refrigerator, and concluded that a low temperature drive with high efficiency can be achieved within a multistage thermoacoustic engine. The concept of Hasegawa et al. was practically realized in this study. Accordingly, a doubleloop-type TWTR driven by a multistage TWTE was constructed. Three etched stainless steel mesh regenerators were installed close to the sweet spot within the prime mover loop and one regenerator was fixed in the refrigerator loop. The onset temperature, the gas oscillations, the refrigeration, and the total COP were measured. The objective was to reduce the onset temperature for thermoacoustic oscillations and achieve a refrigerator temperature of $-100{ }^{\circ} \mathrm{C}$ by placing multiple regenerators close to the sweet spot. 


\section{Experimental setup}

Figure 1 and 2 show the experimental apparatus and its schematic diagram. The apparatus is composed of a prime mover loop, a refrigerator loop, and a branched tube composed of cylindrical stainless steel tubes with an inner diameter of $40 \mathrm{~mm}$. The regenerators used in the apparatus are labeled Regenerator1, Regenerator2, Regenerator3, and Regenerator4, as shown in Fig. 2. All of the regenerators were constructed based on the numerical optimized values. The optimized values of the diameters of regenerators $1,2,3$, and 4 were $0.2,0.2,0.3$, and $0.2 \mathrm{~mm}$, respectively. Regenerator1, Regenerator2, and Regenerator3 have the same axial length of $30 \mathrm{~mm}$ and were installed at optimized positions close to the sweet spot near the T-junction within the prime mover loop. An ambient heat exchanger and a hot heat exchanger were placed on either side of each regenerator. In the refrigerator loop, Regenerator4 (120 mm in length) was sandwiched between the cold and ambient heat exchangers. Here, we use $T_{H}, T_{R}$, and $T_{C}$ to denote the temperatures of the hot heat exchanger, ambient heat exchanger, and refrigerator, respectively.

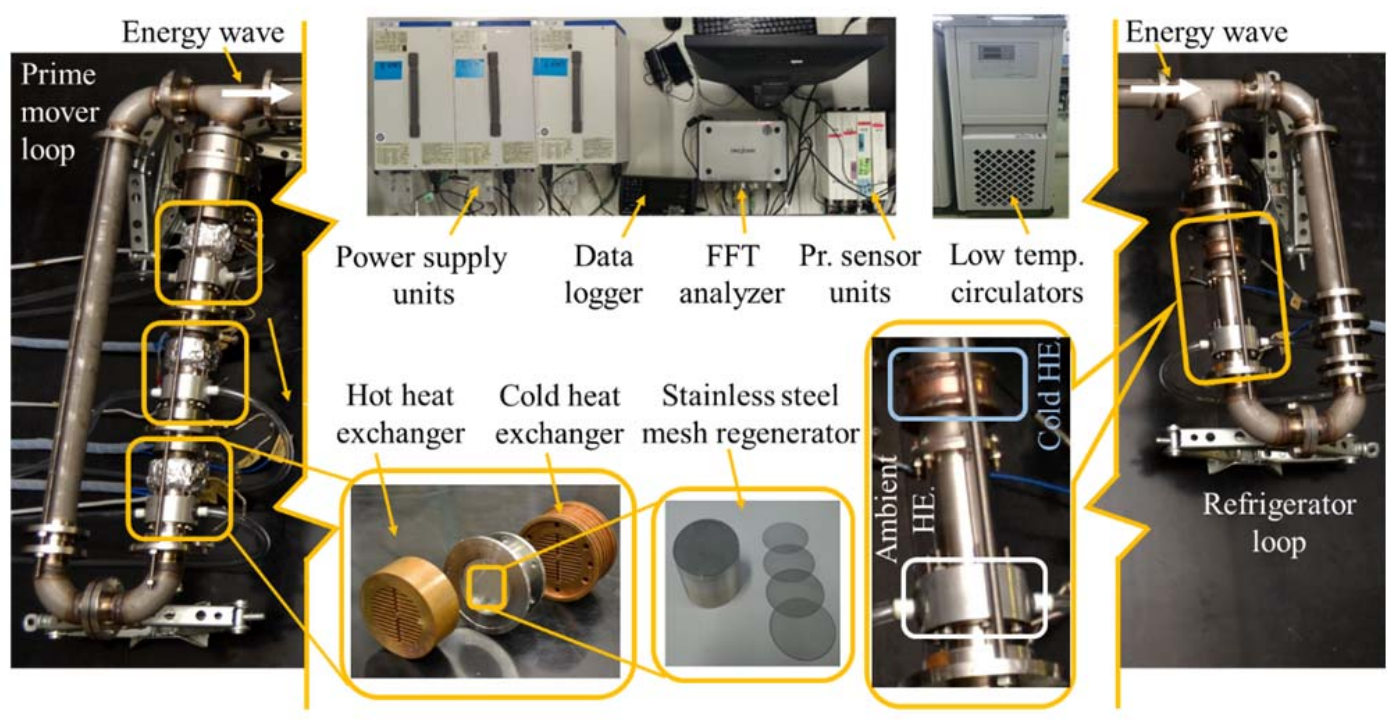

FIG. 1. Experimental setup. 


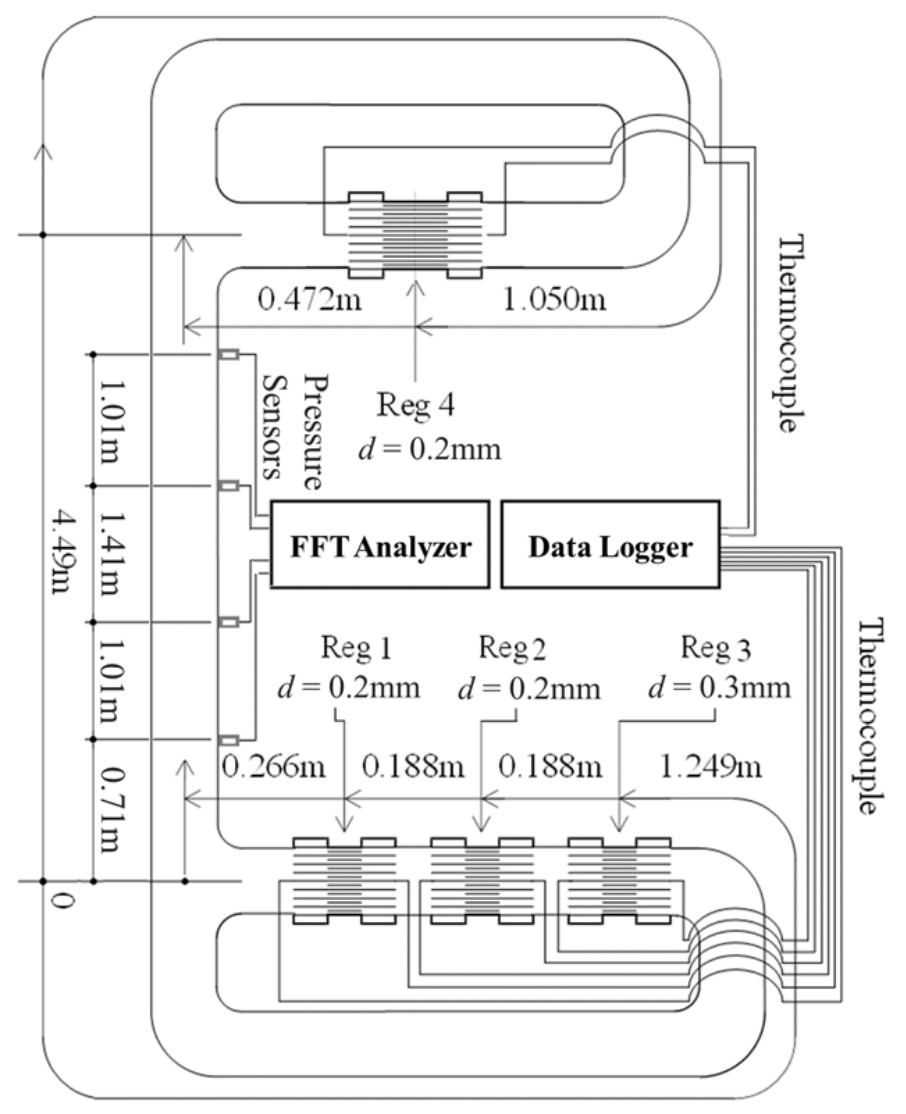

FIG. 2. Schematic diagram of the experimental apparatus.

Etched stainless steel mesh regenerators were used and to reduce the axial thermal conductivity, plate sheets with a $0.1 \mathrm{~mm}$ gap were used along the axial direction, as shown in Fig. 3.

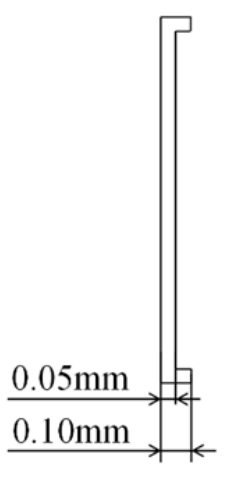

side view

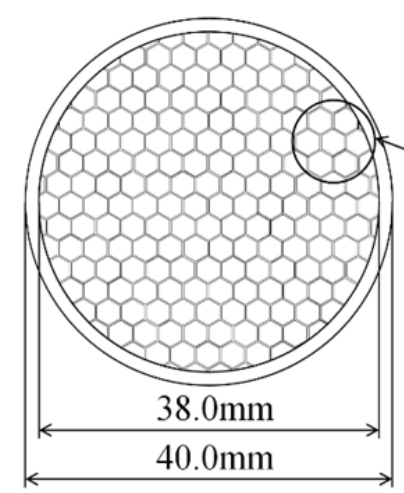

front view

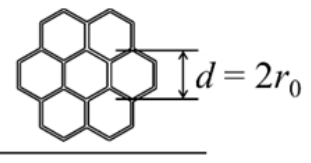

Partial enlarged view
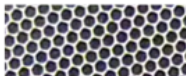
image

FIG. 3. Schematic diagram of an etched stainless steel mesh regenerator.

The heat exchangers consisted of pairs of copper plates aligned in parallel with a 2.0-mm gap between each plate. Each plate was $1.0 \mathrm{~mm}$ thick and $27.0 \mathrm{~mm}$ in axial length, as shown in Fig 4. 


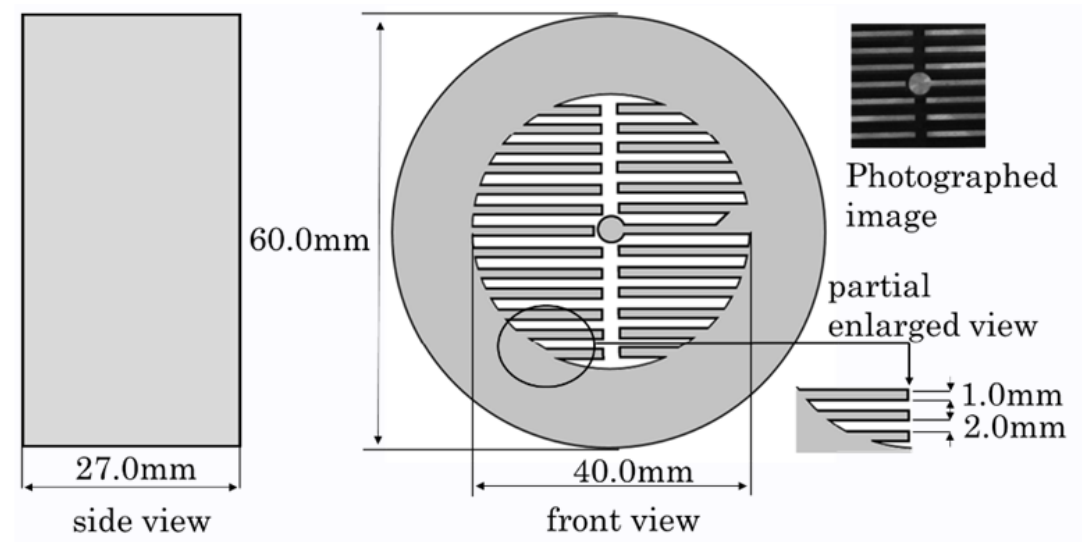

FIG. 4. Schematic diagram of a heat exchanger.

To control the temperature, an electrical heater (2M-1-300) was wound around each hot heat exchanger, whereas water chillers (LTC-1200A) whose uncertainty was $\pm 2.0^{\circ} \mathrm{C}$, were used to keep the temperature of the ambient heat exchangers at room temperature $\left(10^{\circ} \mathrm{C}\right)$. The three hot heat exchangers were always kept at identical temperature during the test. K-type thermocouples with uncertainty range of $\pm 2.5^{\circ} \mathrm{C}$, were used to monitor the temperature of the heat exchangers. The output signals from the thermocouples were recorded by a data logger (GL7000). The pressure transducers were connected to the FFT analyzer (DS-3204) for measuring frequency and to monitor the pressure signals, subsequently the two sensor method ${ }^{20,21}$ was used to measure the pressure amplitude, the velocity amplitude, and the acoustic power. To reduce thermal losses, the hot and cold heat exchangers were insulated with glass wool insulation. A flexible membrane was used in both T-junctions to suppress Gedoen streaming. The models and the companies for the components are listed in Table 1.

Table 1. The companies and the models of components

\begin{tabular}{|l|l|l|}
\hline Components & \multicolumn{1}{|c|}{ Models } & Companies \\
\hline Thermocouples & T-35 (Type-K) & SAKAGUCHI E.H VOC CORP \\
\hline Pressure transducers & PMS-5M-2-1M & JTEKT CORPORATION \\
\hline Power supply & PWR800M & KIKUSUI \\
\hline Low Temp Circulators & LTC-1200A & ASONE CORPORATION \\
\hline Heaters & 2M-1-300 & SAKAGUCHI E.H VOC CORP \\
\hline Data logger & GL7000 & GRAPHTEC \\
\hline FFT Analyzer & DS-3204 & ONO SOKKI \\
\hline
\end{tabular}

\section{Results}

Thermoacoustic energy conversion is essentially controlled by two parameters: the ratio between the radius of the flow channel $r_{0}$ and the thermal penetration depth $\delta_{\kappa}\left(r_{0} / \delta_{\kappa}\right)$ and the phase difference $\Phi$ between the pressure and the cross-sectional mean velocity. When $\Phi$ is close to zero and $r_{0}<<\delta_{\kappa}$, the gas can operate with reversible processes. When the value of $z$ is sufficiently large, viscous losses can be reduced ${ }^{1,2,22}$. For the present apparatus, the calculated parameter $r_{0} / \delta_{\kappa}$ of Regenerator1, Regenerator2, Regenerator3, and Regenerator4 were $0.19,0.19,0.29$, and 0.51 respectively. The obtained $\delta_{\kappa}$ values were much bigger than radius of the flow channel $r_{0}$ and the gap of the 
regenerators. Furthermore, around the sweet spot, $\Phi$ was close to zero and the $z$ value was sufficiently large to reduce viscous losses in the regenerators. Thus, the present apparatus could operate as a TWTR where the working gas passing through the regenerators relies on reversible processes with a low level of viscous losses.

In this work, the diameters and positions of the regenerators were numerically optimized based on the Hasegawa et al. ${ }^{19}$ numerical model. A TWTR was constructed based on the numerically optimized values of the diameters and positions of the regenerators. The working gas with lower value of Prandtl number generally improves the performance of thermoacoustic devices. Since the Prandtl number can be decreased by using a mixture of helium and argon ${ }^{7,23}$, we used a mixture of helium and argon as a working gas. The ratio of helium to argon was 7:3 at 1.0 MPa with an operating frequency of $34.6 \mathrm{~Hz}$. Etched stainless steel mesh regenerators were installed in the sweet spot within the prime mover loop and the refrigerator loop. To reduce the axial thermal conductivity, the plate sheets of the regenerator were constructed with a $0.1 \mathrm{~mm}$ gap along the axial direction. A temperature gradient was generated along the length of each regenerator by external sources that heated one end and cooled the other, and gas oscillations occurred when the hot temperature of the regenerator in the prime mover loop exceeded $85^{\circ} \mathrm{C}$. To assess the behavior of the refrigerator, the temperature of the hot heat exchanger in the primer loop $T_{H}$ as a function of the temperature of the cold heat exchanger in the refrigerator loop $T_{C}$ is shown in Fig. 5.

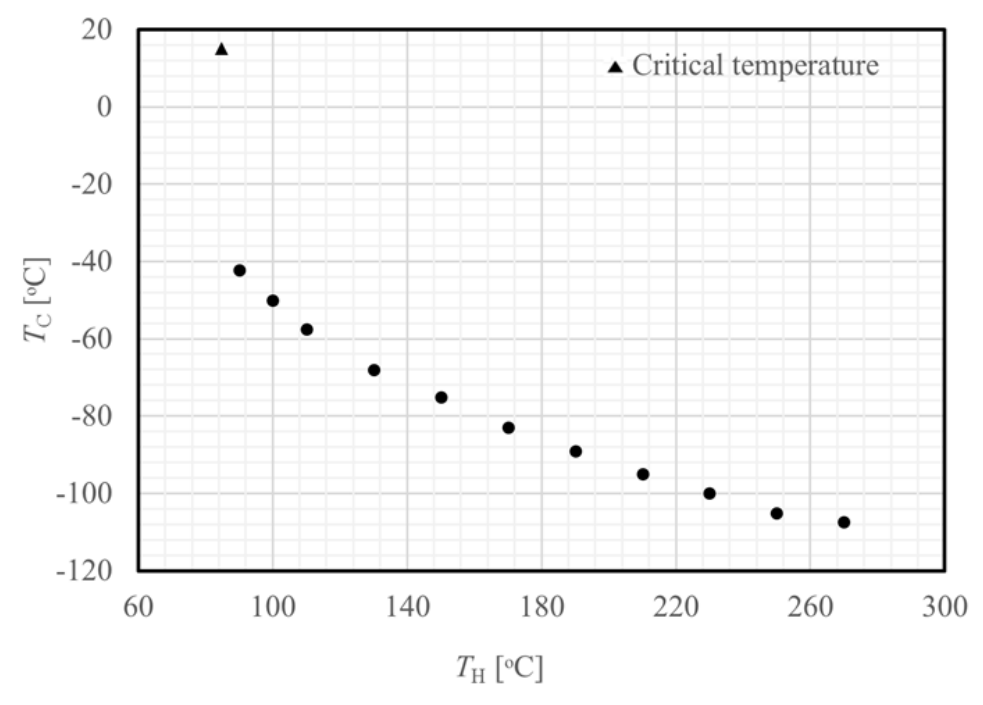

FIG. 5. $T_{C}$ as a function of $T_{H}$.

$T_{H}$ was increased and the value of $T_{C}$ was recorded. The gas oscillations occurred when the hot temperature of the regenerator in the prime mover loop exceeded $85^{\circ} \mathrm{C}$, which is indicated as the critical temperature in Fig 5 . When the hot heat exchanger temperature reached $T_{H}=90{ }^{\circ} \mathrm{C}$, the cold heat exchanger temperature was $T_{C}=-42.3{ }^{\circ} \mathrm{C}$. This means that refrigeration was obtained with a temperature lower than the boiling point of water. Furthermore, $T_{C}$ decreased as $T_{H}$ increased. As a result, $T_{C}=-107.4{ }^{\circ} \mathrm{C}$ when $T_{H}=270{ }^{\circ} \mathrm{C}$. Consequently, the performance of the refrigerator showed significant improvement in onset temperature, refrigeration and achieving minimum temperature compared with those of the refrigerators reported in previously studies ${ }^{7-9}$. The behavior of the refrigerator can be further analyzed by representing the hot and the cold temperatures curves as function of time, as shown in figure 6 . 


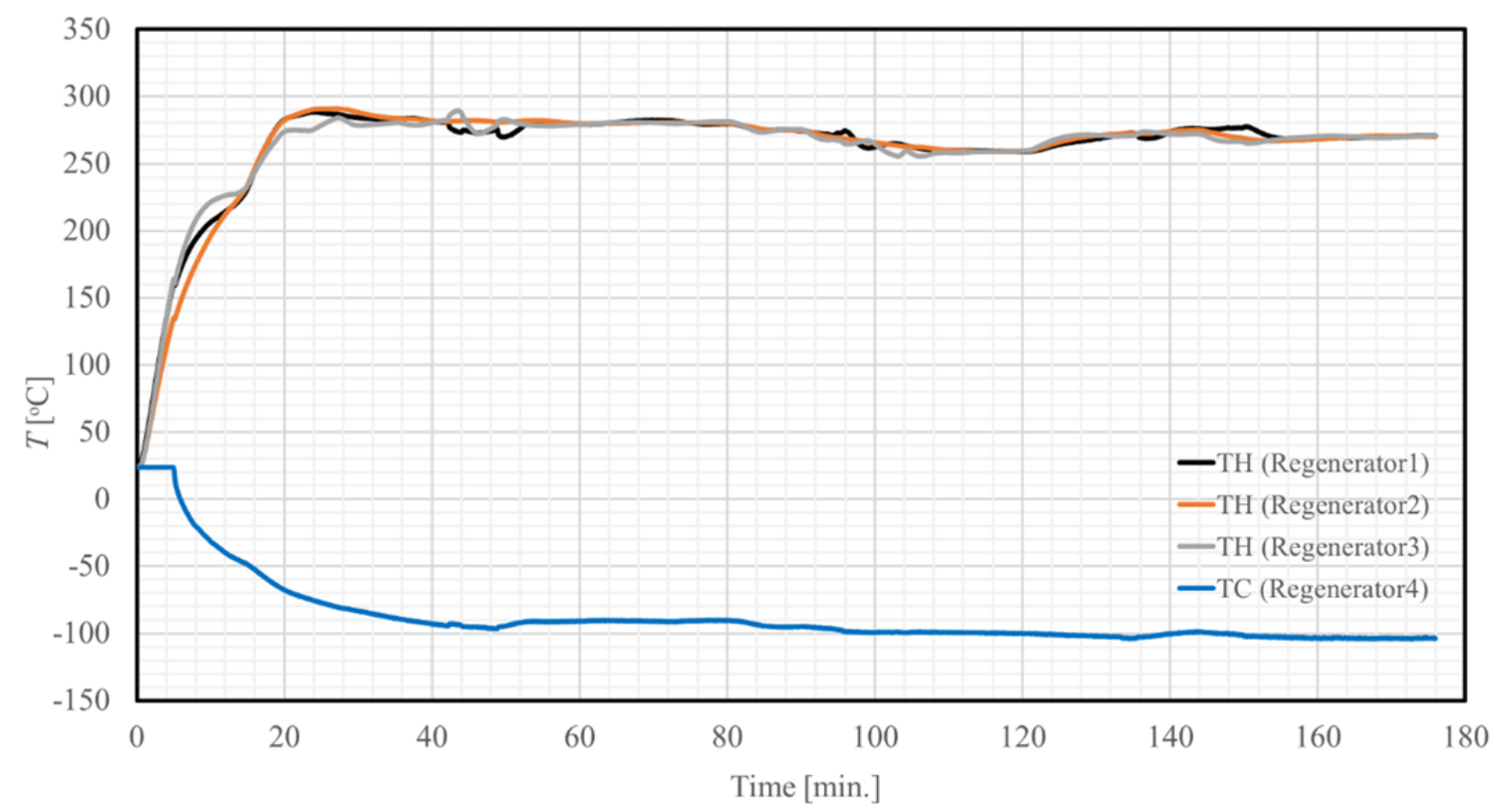

FIG. 6. $T_{C}$ and $T_{H}$ as a function of time.

As can be seen from the figure 6, initially no acoustic wave was present in the system, and the $T_{C}$ remained unchanged at ambient temperature. When the hot temperature of regenerators in the prime mover loop exceeded $150{ }^{\circ} \mathrm{C}$ (time $=5.03 \mathrm{~min}$ ), the acoustic oscillation was excited in the system, the gas parcels began to oscillate, and the heat flow started pumping from the prime mover loop toward the refrigerator loop. As a results, the $T_{C}$ fell rapidly for almost 40 minutes. Subsequently both hot and cold temperatures reached in steady state, and at $T_{H}=270^{\circ} \mathrm{C}$ the minimum temperature of $-107.4^{\circ} \mathrm{C}$ was achieved. The data presented in Fig.6 were recorded in a short period of time (almost 3 hrs.), therefore, a small delay in the onset temperature was observed when compared with the result shown in Fig. 5 However, the last parts of results in both figures (5 and 6) were in good agreement.

The performance of the whole system is expressed in the term of the coefficient of performance (COP), and it is defined as the ratio of the cooling power $\left(Q_{\text {out }}\right)$ to the total sum of input heating power of each engine $\left(Q_{\text {in } 1}+Q_{\text {in } 2}+Q_{\text {in }}\right.$ 3). An electrical heater was placed at the cold heat exchanger in the refrigerator loop and cooling power $\left(Q_{\text {out }}\right)$ was measured as the heat load into the cold heat exchanger. Simultaneously, the heating power $\left(Q_{\text {in } 1}+Q_{\text {in } 2}+Q_{\text {in 3 }}\right)$ was calculated by the sum of the measured electric powers supplied to the three hot heat exchangers within the prime mover loop.

$$
C O P=\frac{Q_{\text {out }}}{Q_{\text {in } 1}+Q_{\text {in } 2}+Q_{\text {in } 3}}
$$




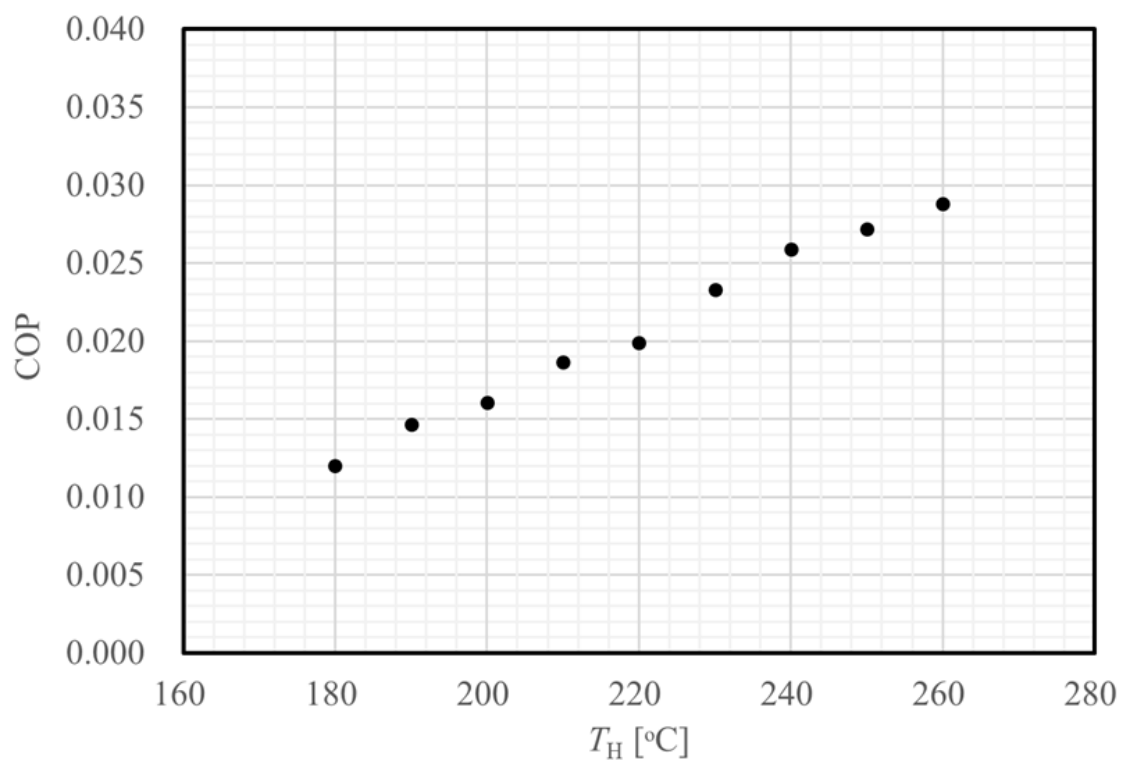

FIG. 7. $C O P$ as a function of $T_{H}$.

Figure 7 illustrates the measured COP as a function of the temperature of hot heat exchangers in the primer loop and it can be seen that the COP of the refrigerator increased gradually as the temperature of hot heat exchangers in the primer loop increased. To avoid the rupture of the membrane due the strong acoustic oscillation at T-junctions of the refrigerator loop, the total COP was measured at $-50{ }^{\circ} \mathrm{C}$. Accordingly, the maximum measured COP was 0.029 when $T_{H}=260{ }^{\circ} \mathrm{C}$, and the corresponding cooling power was $35.6 \mathrm{~W}$.

In summary, the constructed thermoacoustic refrigerator produced gas oscillations and refrigeration at a temperature lower than the boiling point of water. In the vicinity of the sweet spot, $\Phi$ was close to zero, so the $z$ value was sufficiently high and $r_{0}$ values were much less than the $\delta_{\kappa}$ values. Consequently, the refrigerator achieved a minimum cold temperature of $T_{C}=-107.4{ }^{\circ} \mathrm{C}$ when the hot temperature was $T_{H}=270{ }^{\circ} \mathrm{C}$.

\section{Conclusions}

A double-loop-type TWTR driven by a multistage TWTE was constructed. Three etched stainless steel mesh regenerators were installed close to the sweet spot within the prime mover loop and one regenerator was fixed in the refrigerator loop. Consequently, the refrigerator produced the gas oscillations and refrigeration at a temperature lower than the boiling point of water. The evaluated total COP at $-50 \mathrm{C}$ was 0.029 . Furthermore, the refrigerator achieved a minimum cold temperature of $T_{C}=-107.4{ }^{\circ} \mathrm{C}$ when the hot temperature was $T_{H}=270{ }^{\circ} \mathrm{C}$. We conclude that installation of multiple regenerators in the vicinity of the sweet spot of the prime mover loop reduces the onset temperature of thermoacoustic oscillations and improves the cooling performance. We believe that this is a major step towards the development of traveling-wave thermoacoustic engines. 


\section{Acknowledgments}

This study was financially supported by the Advanced Low Carbon Technology Research and Development Program (ALCA) (Grant no. 13414425) of the Japan Science and Technology Agency.

\section{References}

${ }^{1}$ P. H. Ceperley, A pistonless Stirling engine-the traveling wave heat engine, J. Acoust. Soc. Am. 66, 1508 (1979).

${ }^{2}$ T. Yazaki, A. Iwata, T. Maekawa, and A. Tominaga, Traveling wave thermoacoustic engine in a looped tube, Phys. Rev. Lett. 81, 3128 (1998).

${ }^{3}$ S. Backhaus and G. W. Swift, A thermoacoustic Stirling heat engine, Nature 399, 335 (1999).

${ }^{4}$ D. L. Gardner, and G. W. Swift, A cascade thermoacoustic engine, J. Acoust. Soc. Am. 114, 1905 (2003).

${ }^{5}$ G. W. Swift, D. L. Gardner, and S. Backhaus, Acoustic recovery of lost power in pulse tube refrigerators, J. Acoust. Soc. Am. 105, 711 (1999).

${ }^{6}$ T. Yazaki, T. Biwa, and A. Tominaga, Appl., A pistonless Stirling cooler, Phys. Lett. 80, 157 (2002).

${ }^{7}$ Y. Ueda, T. Biwa, and U. Mizutani, Experimental studies of a thermoacoustic Stirling prime mover and its application to a cooler, J. Acoust. Soc. Am. 115, 1134 (2004).

${ }^{8}$ E. Luo, W. Dai, Y. Zhang, and H. Ling, Thermoacoustically driven refrigerator with double thermoacoustic-Stirling cycles, Appl. Phys. Lett. 88, 074102 (2006).

${ }^{9}$ B. Yu, E. Luo, S. F. Li, W. Dai, Z. H. Wua, Experimental study of a thermoacoustically-driven traveling wave thermoacoustic refrigerator, Cryogenics. 51, 49 (2011).

${ }^{10}$ Y. Ueda, C. Kato, Stability analysis of thermally induced spontaneous gas oscillations in straight and looped tubes, J. Acoust. Soc. Am. 124, 851 (2008).

${ }^{11}$ HF. Kang, G. Zhou, Q. Li, Heat driven thermoacoustic cooler based on travelling-standing wave, Energ. Convers. Manag. 51, 2103 (2010).

${ }^{12} \mathrm{ZB}$ Yu, AJ Jaworski, AS Abduljalil, Fishbone-like instability in a looped-tube thermoacoustic engine, J. Acoust. Soc. Am. 128, 188 (2010).

${ }^{13}$ AS Abduljalil, ZB Yu, AJ Jaworski, Design and experimental validation of looped-tube thermoacoustic engine, J. Therm. Sci. 20, 423 (2011).

${ }^{14}$ AS Abduljalil, ZB Yu, AJ Jaworski, Selection and experimental evaluation of low-cost porous materials for regenerator applications in thermoacoustic engines, Materials and Design, 32, 217 (2011).

${ }^{15}$ Y. Nakano, SI. Sakamoto, Study on thermoacoustic system to drive by low temperature: effects of loop-tube thermoacoustic system connected with parallel double stacks on the onset temperature ratio, J. Acoust. Soc. Am. 133, 3232 (2013). 
${ }^{16}$ HF. Kang, F. Jiang, HF. Zheng, AJ. Jaworski, Thermoacoustic travelling-wave cooler driven by a cascade thermoacoustic engine, Appl. Therm. Eng. 59, 223 (2013).

${ }^{17}$ T. Jin, J. Huang, Y. Feng, R. Yang, K. Tang, R. Radebaugh, Thermoacoustic prime movers and refrigerators: thermally powered engines without moving components, Energy, 93, 828 (2015).

${ }^{18}$ T. Biwa, D. Hasegawa, and T. Yazaki, Low temperature differential thermoacoustic Stirling engine, Appl. Phys. Lett. 97, 034102 (2010).

${ }^{19}$ S. Hasegawa, T. Yamaguchi, and Y. Oshinoya, A thermoacoustic refrigerator driven by a low temperature differential, high-efficiency multistage thermoacoustic engine, Appl. Thermal Eng. 58, 394 (2013).

${ }^{20}$ A. M. Fusco, W.C. Ward, G, W. Swift, Two-sensor power measurements in lossy ducts, Journal of the Acoustical Society of America 91, 2229 (1992).

${ }^{21}$ T. Biwa, Y. Tashiro, H. Nomura, Y. Ueda, T. Yazaki, Experimental verification of a two-sensor acoustic intensity measurement in lossy ducts, Journal of the Acoustical Society of America 124, 1584 (2008)

${ }^{22}$ M. M. Bassem, Y. Ueda, and A. Akisawa, Thermoacoustic Stirling heat pump working as a heater, Appl. Phys. Express. 4, 107301 (2011).

${ }^{23}$ J. R. Belcher, W. V. Slaton, R. Raspet, H. E. Bass, and J. Lightfoot, Working gases in thermoacoustic engines, J. Acoust. Soc. Am. 105, 2677 (1999). 